\title{
FILE PRINTING FOR PEDESTRIANS
}

ZOHREH PARSA

High Energy Facilities

BROOKHAVEN NATIONAL LABORATORY

Associated Universities

Upton, New York 11973

[November 1985]

This note is written to accomodate users of Accelerator Physics Data Base BNLDAG: : DUAO: [PARSA1], and BNLCLn: : \$2\$DUA7: [ZOHREH], (n=1,2,3 or 4), etc. It describes different ways of printing files and more. [Note that, due to CYBERVAX revisions, the node Id's have been changed to 3 digits, described as yyy in this paper]. 
FOLLOWING ARE THE COMMANDS THAT CAN BE USED TO PRINT [VAX OR CDC] FILES ON BNL [VAX OR CDC] PRINTERS:

Note: To print more than one copy, use the /COPIES-n $(n=$ any number of copies from 1 to 255).

For example;

\section{$\$$ PRINT/COPIES $=5$ SYNCH.FOR; 3}

will print 5 copies of the file SYNCH.FOR; 3 .

a. TO PRINT FILES ON BNLCLUSTER [BNLCL2]:

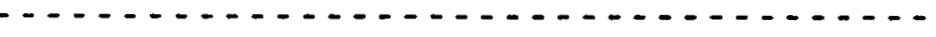

(NOTE: The following commands must be

given from the BNLCLUSTER;

Printers are in the Computer Center BIdg.515)
a. $\$$ PRINT FILE.EXT;2
b. $\$$ PRINTNL FILE.EXT;2
c. \$ PRINTL FILE.EXT;2
d. \$VP FILE.EXT; 2
e. $\$$ PRINT/DEVICE=LVAO
f. $\$$ PRINT/DEVICE=TXAI
g. $\$$ PRINT/DEVICE $=$ LPSI5

[this will print at the center]

[this will print on unruled paper]

[this will print on ruled paper]

[this will print on VERSATEC printer]

[same as d above]

[prints on the center Lazer printer]

[prints on LP515 printer at the center]

b: TO PRINT A FILE ON BNLDAG PRINTERS:

(Printers are in building 940)

1. $\$$ PRINT FILE.EXT;2 [this will print a copy of file.ext on the VERSATEC printer SYS\$PRINT LPAO: in bIdg. 940]

2. \$ COPY FILE.EXT;2 LPAO: <return>

[This will print a copy of file.ext; 2 on LPAO PRINTER., same as above]

3. COPY FILE.EXT;2 BNLHEP::IPAO: <return>

[This will print a copy of

file.ext; 2 on VERSATEC

printer SYSSPRINT LPAO:

4. $\$$ COPY <return>

from: FIIE.EXT;2 <return>

to: BNL734::LPA0: [This will print a copy

of file.ext;2 on $6001 \mathrm{pm}$

impact printer LPAO:] 


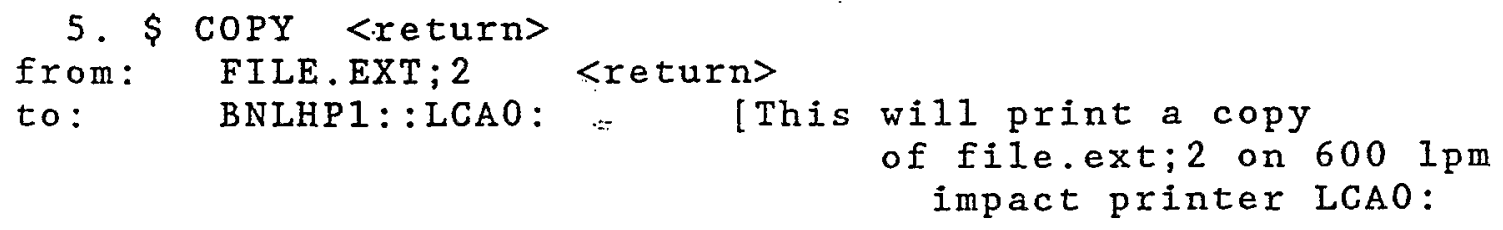

$* * * * * * * * * * * * * * * * * * * * * * * * * * * * * * * * * * * * *$

c. 6. To print a program that is on BNLDAG (e.g. from DUAO: [PARSA1.SYNCH] directory), on MFA. XX PRINTERS, where $x x$ is the printers ID. For example;

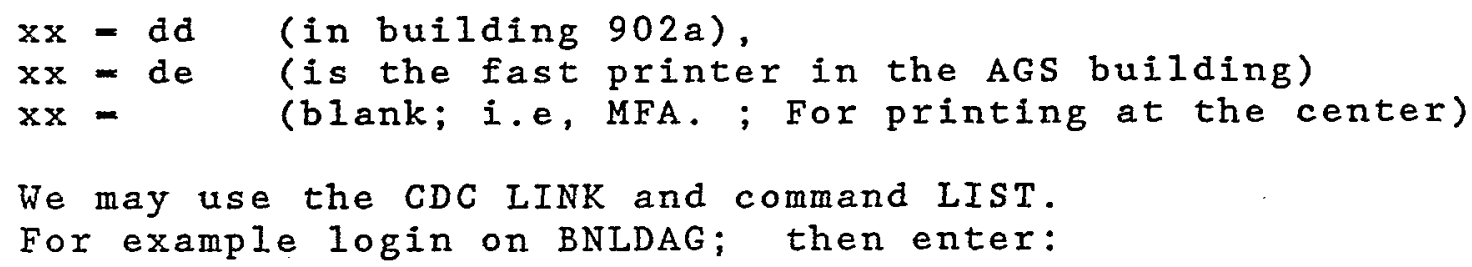

Note, ANAME is the header name for the printed copy.

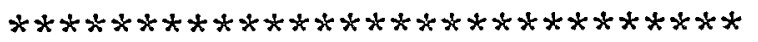

d. We may also use the following command (cyber7600), and print a VAX file on the MFAxx, (e.g. MFA.) printer:

1. After the VAX \$ prompt, use an editor (e.g. EDT) and create a file (example \$EDIT VAX.FILE) containing the following :

ANAME, STMFZ. any comment you like ACCOUNT,parsa1,123,123. ! [parsa1=yourname, 123=yourno.] GETPF (LFN, $\$ v a x-f i l e \$, S T=C V X y y y, I D=v a x u s e r n a m e, P W=v a x-p a s s w d, C V=A V F)$. DISPOSE (LFN, *PR, ST-MFAIXX

LFN $=$ logical file name

yyy = node ID; [use show net to get this information] where your vax-file; to be printed resides; E.G.; $;$ bnldag ID $=030$ BNLCLI ID $=011$ BNLCL2, 3,4 ID $=012,013,014$ respectively.

$\mathrm{xx}=$ printer ID, same as in above; E.g. dd, i.e. MFA.DD 
2. enter $\$$ MSUB VAX.FILE <return>.

$* * * * * * * * * * * * * * * * * * * * * * * * * * * * * * * * * * * *$

e. Finally, we may use the following to print a CDC file on a BNLCLUSTER printers:

[after the VAX $\$$ prompt enter:]

1. Edit a file which contains the following CDC commands.

i)

ANAME, STMFZ. $\quad 7600$ permanent file

ACCOUNT, PARSA1.123,123.

attach, ifn, permanentfile, id=name.

SAVEPF, LFN, \$VAX-FILE, ST-CVXYYy, ID-vaxusername, PW-vax-passwd, CV-AVF.

q zeof

aname,stmfz. $\quad 6600$ permanent file

account, parsa1,123,123.

attach, Ifn, permanentfilenam, id=name, st=stp.

rewind, Ifn.

copy, $\operatorname{lfn}, 1$ fnl.

rewind, Ifnl.

savepf, Ifnl, $\$ v a x-f i l e \$, s t=c v x y y y, i d=v a x u s e r n a m e, p w=v a x-p a s s w o r d, c v=a v f$ q $q$ e of

LFN = logical file name

yyy - node ID; [use show net to get this information] where your vax-file; to be printed resides;

For example:

$B N L D A G I D=030$

BNLCL1 ID -011

BNLCL2, 3,4 ID $=012,013,014$ respective1y.

$\mathrm{xx}$ - printer ID, same as in above;

E.g. dd, i.e. MFA.DD

2. After creating the above VAX file, [e.g. called CDC.FILE which includes i) or ii)] enter:

\$ MSUB CDC.FILE <return> 
3. and then,

\$ PRINT/DEVICE=printername FILE.EXE

!where FILE.EXE is the vax-file, that was brought from $C D C$ in step 2 .

$* * * * * * * * * * * * * * * * * * * * * * * * * * * * * * * *$

HOW TO STOP A JOB:

1. TO DELETE A PRINT JOB:

i. To delete the currently printed job:

$\$$ STOP/ABORT LPAO:

ii. To delete a waiting print job:

$\$$ DELETE/ENTRY=JOB LPAO:

2. To check and fix the printer (e.g. change paper) \$ STOP/QUEUE/NEXT LPAO:

3. To delete the job [on BNLDAG, or with operator priviledge on BNLCLUSTERS]

$$
\begin{aligned}
& \$ \text { DELETE/QUEUE LPAO (to delete job) } \\
& \$ \text { STOP/QUEUE LPAO (to change paper) } \\
& \$ \text { START/QUEUE LPAO }
\end{aligned}
$$

4. TO STOP A CURRENTLY EXECUTING JOB:

\$ STOP/QUEUE/ENTRY-JOB SYS\$BATCH ;Or

\$ DELETE/ENTRY-JOB SYS\$BATCH

5. To get the JOB, use:

$\$$ SHOW QUEUE ; [this shows all YOUR job in aII QUEUES]

$\$$ SHOW QUEUE/ALL

$\$$ SHOW QUEUE SYS\$BATCH

\$ SHOW QUEUE SYS\$PRINT 